\title{
Resultados del trasplante pulmonar en pacientes en situación de urgencia
}

\author{
JOEL MELO T.***, MARÍA T. PARADA C.**, CLAUDIA SEPÚLVEDA L.*, \\ VIRGINIA LINACRE S.*, MAURICIO SALINAS F.*, DAVID LAZO P.**, \\ ELI VILLALABEITÍA R.** y JOSÉ M. CLAVERO R.**
}

\section{Results of lung transplant patients in emergency situation}

Introduction: In Chile, a number of criteria were agreed for emergency lung transplant in order to diminish the mortality of candidates on the waiting list. Objetive: To evaluate short-term transplant patients in emergency condition. Methodology: Retrospective analysis of medical records of patients transplanted from January 2012 to July 2015 demographic data, underlying disease, early and late complication, and survival were recorded. Results: Out of 59 patients transplanted in this period, 18 have been in an emergency condition. Underlying pulmonary disease were: pulmonary fibrosis $(n=13)$, cystic fibrosis $(n=3)$, bronchiolitis obliterans (1) and pulmonary hypertension (1). The dependence of non invasive mechanical ventilation was the main reason for urgency (89\%). $76 \%$ required intraoperative extracorporeal support. Survival at 30 days and 12 months was 94 and 87\% respectively. Conclusion: Lung transplantation is a short-term emergency procedure with good results in survival

Key words: Lung transplant, emergency, survival.

\section{Resumen}

Introducción: En Chile se consensuaron una serie de criterios de urgencia para trasplante pulmonar con el fin de disminuir la mortalidad de candidatos en lista de espera. Objetivo: Evaluar la sobrevida a corto plazo de pacientes trasplantados en condición de urgencia. Metodología: Análisis retrospectivo de fichas clínicas de pacientes trasplantados desde enero del 2012 a julio del 2015. Se consignó datos demográficos, enfermedad de base, complicaciones precoces, tardías y sobrevida. Resultados: De 59 pacientes trasplantados en este periodo 18 han sido en urgencia. Enfermedad de base: fibrosis pulmonar $(n=13)$, fibrosis quística $(n=3)$, bronquiolitis obliterante $(n=1)$, hipertensión pulmonar $(n=1)$. La dependencia de ventilación mecánica no invasiva fue el principal motivo de urgencia (89\%). Un 76\% requirió de soporte extracorpóreo intraoperatorio. La sobrevida a 30 días y a 12 meses fue de 94 y 87\% respectivamente. Conclusión: El trasplante pulmonar en situación de urgencia es un procedimiento con buenos resultados en sobrevida a corto plazo.

Palabras clave: Trasplante pulmonar, urgencia, supervivencia.

\section{Introducción}

El trasplante pulmonar (TP) es la única opción de tratamiento para una serie de pacientes seleccionados con enfermedades pulmonares en fase terminal ${ }^{1}$. La escasez de donantes es un problema a nivel mundial reflejándose en la tasa de donantes efectivos que en Chile es de 5,4 donantes pmh (por millón de habitantes), muy por debajo de las tasas de países como Uruguay $(20 \mathrm{pmh})$ y España (34 pmh) (www.trasplante.cl). Distintas estrategias se han implementado para aumentar la reserva de pulmones para donación, entre las cuales se pueden señalar: uso de donantes marginales, donantes en paro cardíaco y trasplante lobar en donante vivo. Sin embargo, estas estrategias no han sido suficientes para frenar el incremento de mortalidad en lista de espera observada a nivel

\footnotetext{
* Instituto Nacional del Tórax, Providencia, RM, Chile.

** Centro de Trasplantes Clínica Las Condes, Las Condes, RM, Chile.
} 
mundial ${ }^{2}$. En países como Francia, Reino Unido e Italia la mortalidad de pacientes en lista de espera es de $8,3 \%, 10 \%$ y $11 \%$ respectivamente ${ }^{3}$.

Distintos métodos de priorización han sido empleados a nivel mundial para disminuir la mortalidad en lista de espera, entre ellas el sistema LAS (Lung Allocation Score) y otros criterios de urgencia acordados en diferentes países. En el año 2011 los distintos centros de trasplante a nivel nacional consensuaron los siguientes criterios de urgencia para TP:

- Paciente incluido previamente en lista de espera al menos 1 mes, más uno de los siguientes criterios:

1) En VMI (ventilación mecánica invasiva) $\mathrm{o}$ en ECMO (oxigenación por membrana extracorpórea).

2) Hospitalizado con VMNI (ventilación mecánica no invasiva) y/u

3) Hospitalizado con elevada concentración de $\mathrm{O}_{2}$ que impida el alta.

- Además, se consideró como condición de urgencia a pacientes hipersensibilizados en tratamiento con más de 6 meses en lista de espera.

Se consideraron como criterios de exclusión de ingreso a urgencia del paciente la presencia de sepsis y falla multiorgánica.

El objetivo de este estudio es evaluar los resultados en sobrevida a corto plazo en pacientes trasplantados de pulmón en situación de urgencia en los dos principales centros clínicos de trasplante a nivel nacional.

\section{Metodología}

Se revisaron retrospectivamente los registros clínicos de pacientes trasplantados de pulmón en situación de urgencia (TU) desde enero de 2012 a julio de 2015 del Instituto Nacional del Tórax (INT) y de la Clínica Las Condes. Se excluyeron de nuestro análisis los trasplantes cardiopulmonares.

Se consignaron características demográficas, tipo de trasplante, motivo de ingreso a lista de urgencia, promedio de días en urgencia al momento de trasplante, uso de ECMO intraoperatorio, complicaciones quirúrgicas y médicas precoces ( $<6$ meses) y tardías, estadía hospitalaria y sobrevida a 30 días, a 1 y 2 años post-trasplante. Además, se compararon los antecedentes con datos similares de pacientes trasplantados en lista convencional (TC) en el mismo período. La indicación de trasplante se basó en las recomenda- ciones de la Sociedad Internacional de Trasplante de Corazón y Pulmón (ISHLT) publicadas en el año $2006^{4}$.

La inmunosupresión incluyó terapia de inducción con Basiliximab y metilprednisolona y de mantención con: prednisona, tacrolimus y micofenolato o azatioprina. Todos los pacientes recibieron profilaxis antiviral con aciclovir ( por 4 a 6 meses) o valganciclovir (en pacientes IgG negativos para $\mathrm{CMV}$ ), antihongos por un año con itraconazol y cotrimoxazol forte trisemanal de mantención. Los exámenes realizados en cada control fueron: exámenes generales, niveles de inmunosupresión, Rx tórax (TAC de tórax según evolución), espirometría y carga viral para CMV. El seguimiento fue semanal el primer mes, quincenal hasta el tercer mes, mensual hasta el año y luego cada 2 o 3 meses o según necesidad.

Los Comités de Ética de ambas instituciones analizaron y aprobaron el estudio.

\section{Análisis estadístico}

Los datos se describen mediante media y desviación estándar para aquellas variables continuas y utilizando frecuencias absolutas y relativas para las categóricas. Para comparar las características entre ambos grupos se utilizó prueba exacta de Fisher o prueba de Wilcoxon según correspondiera; se verificó previamente la normalidad de las variables continuas.

Para el análisis de supervivencia se utilizó el método de Kaplan Meier censurando el seguimiento de todos los pacientes todavía vivos al $1^{\circ}$ de agosto de 2015. Para la comparación de supervivencia entre grupos se utilizó prueba de log rank. Se definió un nivel de significación de 0,05 a dos colas. Los análisis se realizaron en el programa Stata 10.0.

\section{Resultados}

Se han realizado 59 trasplantes en el período señalado de los cuales el $32 \%$ son bipulmonares y el $57,6 \%$ son de género masculino.

Las características demográficas de los pacientes trasplantados de urgencia se muestran en la Tabla 1. Dieciocho pacientes han sido trasplantados con criterios de urgencia $(30 \%$ del total de $\mathrm{TP}), 77 \%$ de género masculino. El promedio de edad fue de 42 años. Trece pacientes recibieron trasplante monopulmonar. El principal diagnóstico de base fue fibrosis pulmonar en 13 pacientes, 3 casos de fibrosis quística (FQ), un paciente con bronquiolitis obliterante secundaria a trasplante de médula ósea y un caso de hipertensión pul- 
monar primaria (HTP) refractaria al tratamiento. La principal indicación de ingreso a urgencia fue el paciente hospitalizado dependiente de VMNI (89\%). Una paciente de 24 años que cursó con falla respiratoria catastrófica secundaria a nitrofurantoína, estuvo 123 días conectada a ECMO antes del trasplante y ha tenido buena evolución post trasplante a la fecha. Además se incluyó un paciente hipersensibilizado con HTP primaria que no respondió a terapia estándar de su enfermedad con más de 6 meses en lista de espera y que requirió trasplante bipulmonar.

Los principales resultados postoperatorios de pacientes trasplantados en situación de urgencia se muestran en la Tabla 2. La mayoría (76\%) requirió soporte extracorpóreo intra-operatorio; 7 casos con ECMO y 7 bypass cardiopulmonar (BCP)

Hubo 6 complicaciones quirúrgicas precoces por sangrado y 1 estenosis bronquial. Las complicaciones médicas precoces fueron 3 rechazos celulares agudos y 9 episodios infecciosos: 4 neumonias y 5 infecciones por CMV (1 neumonitis y 4 viremias). La principal complicación tardía fueron las infecciones con una enfermedad por CMV que causó fallecimiento de un paciente, una neumonía bacteriana, virosis por coronavirus y dos infecciones por virus herpes.

La estadía hospitalaria promedio de TU fue de

Tabla 1. Características demográficas de 18 pacientes trasplantados de pulmón en urgencia

\begin{tabular}{|lc|}
\hline Masculino/femenino (n) & $14 / 4$ \\
Edad promedio/rango (años) & $42(12-64)$ \\
IMC/rango (kg/m²) & $21(17-30)$ \\
Diagnóstico (n) & \\
Fibrosis pulmonar & 13 \\
Fibrosis quística & 3 \\
BOS & 1 \\
Hipertensión pulmonar & 1 \\
Tipo de trasplante & \\
Bipulmonar & 5 \\
Monopulmonar & 13 \\
Tiempo en lista de espera al activar & \\
urgencia (días)/rango & $80(1-259)$ \\
Motivo de urgencia & \\
VMNI & 16 \\
ECMO & 1 \\
HTP refractaria & 1 \\
\hline
\end{tabular}

IMC: Índice de masa corporal; BOS: Bronquiolitis obliterante; VMNI: Ventilación mecánica no invasiva; ECMO: Oxigenación por membrana extracorpórea. HTP: Hipertensión pulmonar.
25 días (rango: 11 a 50 días) y la mortalidad perioperatoria fue de un $5,5 \%$.

La Tabla 3 compara los resultados de pacientes trasplantados en situación de urgencia (TU) con los pacientes trasplantados en forma convencional (TC).

El tiempo promedio de espera para trasplante en pacientes en situación de urgencia fue de 80 días (rango: 1 a 259 días) mientras que en pacientes trasplantados en lista convencional fue de 275 días (rango 9 a 891 ) no alcanzando diferencias estadísticas significativas $(\mathrm{p}=0,15)$. El número de días post-operatorios en VMI promedio que tuvieron los pacientes TU (5,6 $\pm 5,3$ días) fue significativamente mayor $(\mathrm{p}=0,02)$ que en los TC $(2,3 \pm 2,7$ días $)$.

Cinco pacientes (27\%) trasplantados en situación de urgencia han fallecido durante el seguimiento. Las causas de muerte de estos pacientes fueron: sepsis por Pseudomonas sp (día 6), enfermedad por CMV (10 meses), varicela diseminada (21 meses), pancreatitis (20 meses) y neumonía grave (33 meses). Nueve pacientes $(21 \%)$ han fallecido en el grupo de pacientes trasplantados en lista convencional, de los cuales 55\% lo hizo en los primeros 30 días: dos por accidente vascular encefálico, uno por disfunción primaria del injerto, uno por fibrilación ventricular y uno por aspergilosis invasiva.

La supervivencia a 30 días, 1 y 2 años fue de $94,4,87,2$ y $52,3 \%$ en el grupo TU mientras que en los TC fue de $87,8,80,1$ y $77,1 \%$ no encontrándose diferencia estadísticamente significativa entre ambos grupos $(\mathrm{p}=0,486)$ (Figura 1 , Tabla 4).

Tabla 2. Resultado post-quirúrgico de pacientes trasplantados de pulmón en urgencia

\begin{tabular}{lc|}
$\begin{array}{l}\text { Estadía hospitalaria (días)/rango post } \\
\text { trasplante }\end{array}$ & 25 (11-50) \\
$\begin{array}{l}\text { Soporte extracorpóreo } \\
\text { BCP (\%) }\end{array}$ & 38 \\
ECMO (\%) & 38 \\
VM post trasplante (horas promedio/ & $135(6-408)$ \\
rango) & \\
Mortalidad perioperatoria (<30 días) \% & 5,5 \\
Sobrevida (\%) & \\
30 días & 94 \\
1 año & 87 \\
2 año & 52 \\
\hline
\end{tabular}

BCP: Bypass cardiopulmonar; ECMO: Oxigenación por membrana extracorpórea. 
Tabla 3. Comparación de pacientes trasplantados de pulmón en urgencia (TU) y trasplantados en lista convencional (TC)

\begin{tabular}{|c|c|c|c|}
\hline Variables & TU $(n=18)$ & TC $(n=41)$ & Valor de $p$ \\
\hline Masculino/femenino (n) & $14 / 4$ & $20 / 21$ & \\
\hline Edad promedio (años)/DS & $42,9+16,4$ & $47,9+14,9$ & 0,15 \\
\hline \multicolumn{4}{|l|}{ Diagnóstico (n): } \\
\hline Fibrosis pulmonar & 13 & 28 & \\
\hline Fibrosis quística & 3 & 3 & \\
\hline BQ no FQ & 0 & 6 & \\
\hline BOS & 1 & 0 & \\
\hline Hipertensión pulmonar & 1 & 0 & \\
\hline Otras & & 4 & \\
\hline \multicolumn{4}{|l|}{ Tipo de trasplante } \\
\hline Bipulmonar & 5 & 14 & 0,76 \\
\hline Monopulmonar & 13 & 27 & \\
\hline Tiempo en lista de espera (días/DS) & $80,9+89,9$ & $275+301$ & 0,15 \\
\hline VMI (días) & $5,6+5,3$ & $2,3+2,7$ & 0,02 \\
\hline Mortalidad peri-operatoria \% (<30 días) & 5,5 & 12 & \\
\hline
\end{tabular}

BQ: bronquiectasias. FQ: fibrosis quística. BOS: bronquiolitis obliterante.VMI: ventilación mecánica invasiva.

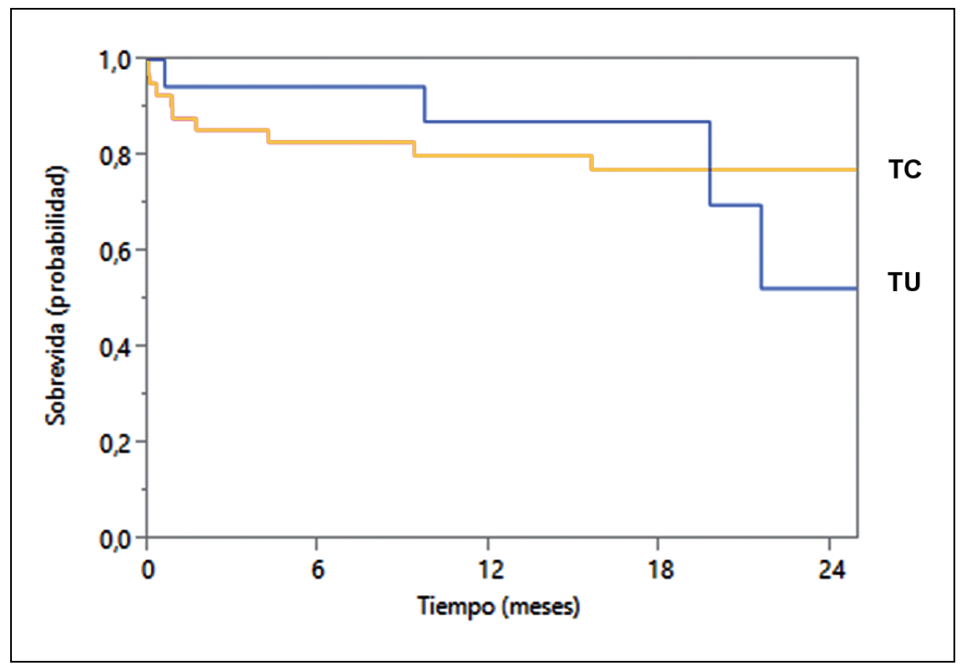

Figura 1. Curvas de supervivencia de trasplantados de pulmón en urgencia (TU) y trasplantados en lista convencional $(\mathrm{TC})(\mathrm{p}=0,486)$.
Tabla 4. Supervivencia de trasplantados de pulmón en urgencia y en lista convencional

\begin{tabular}{|ccc|}
\hline Supervivencia & $\begin{array}{c}\text { Trasplante } \\
\text { urgencia } \\
(\%)\end{array}$ & $\begin{array}{c}\text { Trasplante } \\
\text { convencional } \\
(\%)\end{array}$ \\
\hline 30 días & 94,4 & 87,8 \\
\hline 1 año & 87,2 & 80,1 \\
\hline 2 años & 52,3 & 77,1 \\
\hline
\end{tabular}

\section{Discusión}

El paciente en lista de espera para trasplante pulmonar puede presentar un agravamiento de su enfermedad y requerir ingreso a unidad de cuidados intensivos con alto riesgo de fallecer. La implementación de criterios de urgencia consensuados permite ofrecer posibilidades de trasplante a estos pacientes, en ausencia de contraindicación secundaria a un proceso agudo ${ }^{5}$. 
Existe una creencia de que en los pacientes trasplantados en urgencia, el riesgo de fracaso es alto, planteando un conflicto ético basado sobre todo en que frente a una escasa tasa de donación, el paciente en urgencia pueda fallecer en el trasplante y por lo tanto se pierda un órgano útil para ser trasplantado en otro paciente con más posibilidades de éxito 5 .

A fines del año 2011 los distintos centros de trasplante en conjunto con la Corporación Nacional de Trasplante acordaron una serie de criterios que permitieran priorizar al paciente en lista que presenta un agravamiento $\mathrm{y}$, por lo tanto, tener más opción de recibir un trasplante.

Según información del Instituto de Salud Pública (www.ispch.cl) el 36,4\% de los pacientes trasplantados de pulmón en el año 2012 lo fueron en situación de urgencia, llegando a un $45,5 \%$ en 2014. Sin embargo, no había información hasta ahora del resultado del procedimiento en pacientes trasplantados en esta condición. Este estudio incluye la experiencia de una cohorte de 18 pacientes trasplantados pulmonares en situación de urgencia en los dos principales centros de trasplante a nivel nacional que incluye un centro público y otro privado.

Un punto a considerar en este trabajo es que, si bien, el uso de VMI o ECMO se planteó como criterio de urgencia, es una situación excepcional en nuestro medio y está determinada en gran parte por la experiencia de cada centro. El registro de la ISHLT y varios estudios muestran que la VMI y el ECMO pre-trasplante son factores de riesgo de muerte en el post-trasplante inmediato $^{6,7}$. El uso de VMNI y ECMO despierto (Awake ECMO) pre-trasplante permitió en nuestra serie la rehabilitación muscular y la mejoría nutricional en un grupo de pacientes previo al trasplante. Destaca un caso de una paciente joven de 24 años que esperó 123 días conectada a este soporte la llegada de un órgano compatible y ha tenido una evolución favorable a 18 meses post-trasplante.

La mejoría de los cuidados en las unidades de intensivos y la consolidación de centros con experiencia en ECMO han permitido al paciente en lista de urgencia, esperar un donante mientras se nutre y rehabilita. Creemos que este tipo de soporte jugará un importante rol como puente para trasplante como lo están demostrando publicaciones recientes ${ }^{8}$.

El tiempo promedio en lista de espera del paciente al ser activada la urgencia nacional fue de 80 días (rango 1 a 259 días). Tiempo bastante prolongado al compararlo con lo publicado por otros grupos, en que el tiempo promedio en lista de espera para trasplante de pacientes en urgen- cia es de 6 a 9,8 días ${ }^{9,10}$. El prolongado tiempo de espera de pacientes enlistados de urgencia estuvo dado principalmente por 4 pacientes que esperaron más de 200 días y donde los principales factores causales de demora fueron: pacientes de talla baja que requieren un órgano tamaño compatible y pacientes hipersensibilizados que necesitan estudios de compatibilidad del donante antes del trasplante (cross-match).

Otro punto a destacar es la baja mortalidad peri-operatoria ( $<30$ días) en nuestro grupo, que fue de sólo un 5,5\%, menor que la reportada por Calvo y cols de $36,6 \%$ en un grupo de trasplante español ${ }^{5}$ y del $19 \%$ en la serie francesa de Orsini et $\mathrm{al}^{3}$. Esto probablemente refleja los distintos criterios de urgencia utilizados en cada centro.

En un centro Italiano, Boffini y cols en un reporte de 28 pacientes trasplantados en urgencia encontraron una supervivencia al año de $71,4 \%$, concluyendo que el trasplante en estos pacientes es un procedimiento con resultados aceptables en pacientes seleccionados 9 .

En nuestro estudio, la supervivencia entre los dos grupos (TU y TC) es comparable el primer año, que es el dato más consistente en esta serie analizada (Figura 1 y Tabla 4). A partir de los 24 meses las curvas de sobrevida se separan en forma relevante, con un 52 y $77 \%$ de supervivencia en pacientes trasplantados en situación de urgencia y en forma convencional respectivamente, dato que esta fuertemente influido porque en el grupo de trasplantes en urgencia, la mitad de los pacientes tiene menos de un año de seguimiento y solo tres pacientes tiene más de dos años de trasplante. Lo anterior determina que las muertes y censuras ocurridas a partir del segundo año generen cambios de magnitud relevante en el estimador de Kaplan Meier. En concordancia con lo anterior, en números absolutos ambos grupos tienen porcentaje similar de sujetos fallecidos que fue de $27 \%$ en pacientes trasplantados en situación de urgencia y de $21 \%$ en el grupo de pacientes trasplantados en lista convencional.

Las principales limitaciones de este artículo son el pequeño número de pacientes trasplantados en urgencia y con periodo de seguimiento no mayor a dos años. Sin embargo, es una muestra representativa de nuestra realidad local dado que abarca experiencia de un centro público y uno privado.

Recientemente los distintos centros de trasplante se están reuniendo para reevaluar los criterios de urgencia consensuados en 2011 y analizar eventuales modificaciones en beneficio de los pacientes en lista de espera.

Es necesario evaluar medidas a nivel nacional, 
como revisar la ley de donación, mejorar la información y educación que se entrega a la población general que les permita tomar una decisión informada si desean ser donantes, asociado a medidas de gestión hospitalaria en unidades de urgencia y cuidados intensivos para optimizar el manejo del donante y de nuevas técnicas como perfusión ex vivo o uso de donantes con criterios expandidos que permitan aumentar la reserva de pulmones útiles para trasplante ${ }^{11}$.

\section{Conclusión}

El trasplante pulmonar en situación de urgencia es una opción válida en pacientes seleccionados con una baja mortalidad peri-operatoria y sobrevida a corto plazo comparable con la de pacientes trasplantados en lista convencional.

\section{Bibliografía}

1.- BOFFINI M, RANIERI V M, RINALDI M. Lung transplantation: is it still an experimental procedure? Curr Opin Crit Care 2010; 16: 53-61.

2.- ROMAN A, CALVO V, USSETTI P, BORRO J M. Urgent Lung transplantation in Spain Transplantation Proceedings 2005; 37: 3987-90.

3.- ORSINI B, SAGE E, OLLAND A, COCHET E, TABUTIN M, et al High-emergency waiting list for lung transplantation: early results of a nation-based study European Journal of Cardio-Thoracic Surgery 2014, 46: e41-e47.

4.- ORENS J B, ESTENNE M, ARCASOY S, CONTE J V, CORRIS P, EAGN J J, et al; Pulmonary Scientific
Council of the Internacional Society for Heart and Lung Transplantation: 2006 update-a consensus report from the Pulmonary Scientific Council of tehinternacional Society for Heart and Lung Trasnsplantation. J Heart Lung Transplant 2006; 25: 745-55

5.- CALVO V, PADILLA J, GARCÍA-ZARZAA, BLASCO E, PASTOR J, PARÍS F. El trasplante pulmonar en situación de urgencia Arch Bronconeumol 2003; 39: 111.

6.- CHRISTIE J D, EDWARDS L B, KUCHERYAVAYA A Y, DOBBELS F, KIRK R, RAHMEL A O, et al. The Registry of the International Society for Heart and Lung Trnasplantation: twenty-seventh official adult lung and heart-lung transplant report-2010. J Heart Lung Transplant 2010; 29: 1104-18.

7.- SINGER J P, BLANC P D, HOOPES C, GOLDEN J A, KOFF J L, LEARD L E, et al. The Impact of pretransplant mechanical ventilation on short -and long-term survival after lung transplantation. Am J Transplant 2011; $11: 2197-204$.

8.- DEL SORBO L, BOFFINI M, RINALDI M, RANIERI V M. Bridging to lung transplantation by extracorporeal support. Minerva Anestesiol 2012; 78: 243-50.

9.- BOFFINI M, VENUTA F, REA F, COLLEDAN M, SANTAMBROGIO L, D'ARMINI A M, et al. Urgent lung transplant programme in Italy: analysis of the first 14 months Interactive Cardiovasc Thoracic Surg 2014; 19: 795-800.

10- ROUX A, BEAUMONT-AZUAR L, HAMID A, DE MIRANDA S, GRENET D, BRIEND G, et al. High Emergency Lung Transplantation: dramatic decrease of waiting list death rate without relevant higher posttransplant mortality. Transplant International 2015; 28 : 1092-101.

11- SNELl G, PARASKEVA M, WESTALL G. Donor Selection and Management. Semin Respir Crit Care Med 2013; 34: 361-70.
Correspondencia a:

Dr. Joel Melo T.

Clínica Las Condes

Lo Fontecilla 441

Las Condes, Santiago, Chile.

Email:.jmelo@clinicalascondes.cl 